\title{
Extracting joint weak values from two-dimensional spatial displacements
}

\author{
Hirokazu Kobayashi,, , 田 Graciana Puentes, ${ }^{2, \text { I }}$ and Yutaka Shikano ${ }^{3,4, \text { 团 }}$ \\ ${ }^{1}$ Department of Electronic and Photonic System Engineering, \\ Kochi University of Technology, Tosayamada-cho, Kochi, Japan. \\ ${ }^{2}$ ICFO-Institut de Ciencies Fotoniques, Mediterranean Technology Park, 08860 Castelldefels, Barcelona, Spain \\ ${ }^{3}$ Institute for Molecular Science, Okazaki, Aichi, Japan. \\ ${ }^{4}$ Chapman University, Orange, California, USA.
}

(Dated: August 21, 2018)

\begin{abstract}
The joint weak value is a counterfactual quantity related to quantum correlations and quantum dynamics, which can be retrieved via weak measurements, as initiated by Aharonov and colleagues. In this Rapid Communication, we provide a full analytical extension of the method described by Puentes et al. [Phys. Rev. Lett. 109, 040401 (2012)], to extract the joint weak values of singleparticle operators from two-dimensional spatial displacements of Laguerre-Gauss probe states, for the case of azimuthal index $|l|>1$. This method has a statistical advantage over previous ones since information about the conjugate observable, i.e., the momentum displacement of the probe, is not required. Moreover, we demonstrate that under certain conditions, the joint weak value can be extracted directly from spatial displacements without any additional data processing.
\end{abstract}

PACS numbers: 42.50.Tx, 03.65.Ta, 42.50.Xa

As the well-known examples of the Einstein-PodolskyRosen paradox and the which-path information in the Young double-slit experiment illustrate, quantum dynamics and quantum correlations have eluded our intuitive understanding for a long time. To capture these features, it is important to access the expectation value of products of two observables since such joint observables can contain information about quantum correlations and quantum dynamics. However, in the standard technique, the strong measurement of joint observables is difficult to implement because of the requirement of a nonlinear Hamiltonian [1] and the state reduction resulting from the measurement backaction. In this regard, the joint weak value is a good candidate, as seen in Refs. [2 [5], since this quantity can be experimentally obtained with a tiny measurement backaction from the second-order effects of the time evolution via a local, linear, and singleparticle interaction Hamiltonian.

Historically, the weak value was initiated by Aharonov, Albert, and Vaidman (AAV) [6], inspired from the twotime formulation of the quantum mechanical system [7]. This formulation is characterized by the pre- and postselected states of the system. When a system initially prepared in state $\left|\psi_{\mathrm{i}}\right\rangle$ is post-selected in state $\left|\psi_{\mathrm{f}}\right\rangle$, the weak value of the observable $\hat{A}$ is defined as

$$
\langle\hat{A}\rangle_{\mathrm{w}}:=\frac{\left\langle\psi_{\mathrm{f}}|\hat{A}| \psi_{\mathrm{i}}\right\rangle}{\left\langle\psi_{\mathrm{f}} \mid \psi_{\mathrm{i}}\right\rangle}
$$

which can lie outside the spectrum of $\hat{A}$ and can even take an imaginary number. Recently, there have been various experimental realizations using the weak value

\footnotetext{
*Electronic address: kobayashi.hirokazu@kochi-tech.ac.jp

${ }^{\dagger}$ Electronic address: graciana.puentes@icfo.es

‡Electronic address: yshikano@ims.ac.jp
}

within the foundations of quantum mechanics, for example, in the direct measurement of a wavefunction [8], the which-path measurement in the Young double slit experiment [9], the confirmation of the Heisenberg-Ozawa uncertainty relationship [10], the confirmation of the Hardy paradox [11], the violation of the Leggett-Garg inequality [12], and the measurement of a geometric phase in an interferometer [13].

There are also various proposals for extracting the weak value from experimental data (see for reviews 1416]). Originally, AAV restricted their attention to the standard von Neumann paradigm with a weak interaction Hamiltonian of the form $\hat{H}=g \hat{A} \otimes \hat{P}_{x}$, where $g$ is a small coupling constant and $\hat{P}_{x}$ is the momentum observable of the probe state conjugate to the position observable $\hat{X}$ with $\left[\hat{X}, \hat{P}_{x}\right]=\mathrm{i} \hbar$. Moreover, they assumed that the probe state was initially prepared in a fundamental Gaussian mode. In this case, we can determine the real and imaginary parts of the weak value from the spatial and momentum displacements of the probe state $[6,17$.

Recently, Resch and Steinberg [2] proposed a measurement technique for the weak value of the joint observable as alluded before. They employed a two-dimensional Gaussian probe state and a weak coupling of the system observable $\hat{A}(\hat{B})$ with $x(y)$ dimension of the probe state. By performing a second order expansion in the two-dimensional displacement of the probe state, they showed that it is possible to extract the real part of the joint weak value $\langle\hat{A} \hat{B}\rangle_{\mathrm{w}}$ from the second-order spatial displacement, under the assumption of commuting observables $[\hat{A}, \hat{B}]=0$. This procedure, however, needs the real and imaginary parts of single weak values $\langle\hat{A}\rangle_{\mathrm{w}}$ and $\langle\hat{B}\rangle_{\mathrm{w}}$ to calculate the joint weak value. Thus, we have to obtain full information of the probe wavefunction, i.e., not only the spatial displacement but also the momentum displacement, by taking the Fourier transform of the probe wavefunction. 
In this Rapid Communication, we provide a simple method for extracting the joint weak value only from the spatial displacement of the two-dimensional probe state. This method has a statistical advantage over previous methods since full information of the probe wavefunction is not required. The key idea is to employ LaguerreGauss (LG) modes for probe states, as was initiated by Puentes et al. [3]. We extend this idea and provide the full description of the weak measurement for higher order LG modes with radial index $p=0$ to extract the joint weak values.

The LG modes are given as the natural solutions of the paraxial wave-equation [18] and characterized by a radial index $p$ and an azimuthal index $l$. The modes have zero intensity at its center and an annular intensity distribution. The wavefront of the LG modes is composed of $|l|$ intertwined helical wavefronts, with a handedness given by the sign of $l$. It has been shown that each photon in the LG modes carries a quantized intrinsic orbital angular momentum $l \hbar$, in addition to the spin-like angular momentum $\pm \hbar$ associated with circularly polarized waves [19]. The LG modes have been created using various experimental setups, e.g., using spatial light modulators [20] and using the reflection on a conically-shaped mirror [21]. Furthermore, the LG modes have many applications, for example, for achieving high efficiency of optical tweezers 22], for reducing the thermal noise inside gravitational-wave interferometers 23], and for generating entanglement with high efficiency 24]. The amplitude distribution of the LG modes with radial index $p=0$ is given as

$$
\phi_{i}(x, y)=N\{x+i \cdot \operatorname{sgn}(l) y\}^{|l|} \exp \left(-\frac{x^{2}+y^{2}}{4 \sigma^{2}}\right),
$$

where $\sigma$ is the variance in the case of $l=0, \operatorname{sgn}(\cdot)$ is the sign function, and $N$ is the normalization constant. When $l=0$, Eq. (2) corresponds to a fundamental Gaussian mode. In this case, the amplitude distribution is factorable in two directions, $x$ and $y$. When $|l|>0$, however, it is no longer factorable, and this is a key factor for retrieving the joint weak values.

Consider a weak interaction between the LG probe state $\left|\phi_{\mathrm{i}}\right\rangle=\int \mathrm{d} x \mathrm{~d} y \phi_{\mathrm{i}}(x, y)|x, y\rangle$ and an initial state $\left|\psi_{\mathrm{i}}\right\rangle$ of the system for the joint weak measurement of the observables $\hat{A}$ and $\hat{B}$. The total input state is $\left|\Psi_{\mathrm{i}}\right\rangle=$ $\left|\psi_{\mathrm{i}}\right\rangle \otimes\left|\phi_{\mathrm{i}}\right\rangle$. For the measurement process, we use the standard von Neumann paradigm with the local, linear, and single-particle interaction Hamiltonian as follows:

$$
\hat{H}=g \delta\left(t-t_{0}\right)\left(\hat{A} \otimes \hat{P}_{x}+\hat{B} \otimes \hat{P}_{y}\right),
$$

where a coupling constant $g$ is sufficiently small, and $\hat{P}_{x}$ and $\hat{P}_{y}$ are the momentum observables of the probe conjugate to two commuting position observables $\hat{X}$ and $\hat{Y}$, respectively. Here we have taken the interaction to be impulsive at time $t=t_{0}$ and the same coupling constant $g$ between $x$ and $y$ directions for simplicity.
After the interaction between the system and probe states, we post-select the system in state $\left|\psi_{\mathrm{f}}\right\rangle$, resulting in the probe state:

$$
\left|\phi_{\mathrm{f}}\right\rangle=\left\langle\psi_{\mathrm{f}}\left|\mathrm{e}^{-\mathrm{i} g\left(\hat{A} \otimes \hat{P}_{x}+\hat{B} \otimes \hat{P}_{y}\right) / \hbar}\right| \psi_{\mathrm{i}}\right\rangle\left|\phi_{\mathrm{i}}\right\rangle .
$$

We denote the expectation value of an observable $\hat{M}$ in the initial probe state $\left|\phi_{\mathrm{i}}\right\rangle$ and the final probe state $\left|\phi_{\mathrm{f}}\right\rangle$ as

$$
\langle\hat{M}\rangle_{\mathrm{i}} \equiv \frac{\left\langle\phi_{\mathrm{i}}|\hat{M}| \phi_{\mathrm{i}}\right\rangle}{\left\langle\phi_{\mathrm{i}} \mid \phi_{\mathrm{i}}\right\rangle},\langle\hat{M}\rangle_{\mathrm{f}} \equiv \frac{\left\langle\phi_{\mathrm{f}}|\hat{M}| \phi_{\mathrm{f}}\right\rangle}{\left\langle\phi_{\mathrm{f}} \mid \phi_{\mathrm{f}}\right\rangle}
$$

We can calculate the displacement $\langle\hat{M}\rangle_{\text {fi }}$ by expanding the time evolution operator in Eq. (4) up to the second order in the coupling constant $g$ :

$$
\begin{aligned}
\langle\hat{M}\rangle_{\mathrm{fi}} & \equiv\langle\hat{M}\rangle_{\mathrm{f}}-\langle\hat{M}\rangle_{\mathrm{i}} \\
& \simeq \frac{2 g}{\hbar} \operatorname{Im}\left\langle\hat{M} \hat{H}_{1}\right\rangle_{\mathrm{i}}+\frac{g^{2}}{\hbar^{2}}\left(\left\langle\hat{H}_{1}^{\dagger} \hat{M} \hat{H}_{1}\right\rangle_{\mathrm{i}}-\operatorname{Re}\left\langle\hat{M} \hat{H}_{2}\right\rangle_{\mathrm{i}}\right),
\end{aligned}
$$

where

$$
\begin{aligned}
& \hat{H}_{1} \equiv \hat{P}_{x}\langle\hat{A}\rangle_{\mathrm{w}}+\hat{P}_{y}\langle\hat{B}\rangle_{\mathrm{w}}, \\
& \hat{H}_{2} \equiv \hat{P}_{x}^{2}\left\langle\hat{A}^{2}\right\rangle_{\mathrm{w}}+\hat{P}_{y}^{2}\left\langle\hat{B}^{2}\right\rangle_{\mathrm{w}}+\hat{P}_{x} \hat{P}_{y}\langle\hat{A} \hat{B}+\hat{B} \hat{A}\rangle_{\mathrm{w}} .
\end{aligned}
$$

$\hat{H}_{1}$ and $\hat{H}_{2}$ are respectively the first- and second-order terms of the time evolution operator in the coupling constant $g$. In case of the large weak value, the approximation in Eq. (6) is invalid because of the measurement backaction [25]. In this Rapid Communication, however, we treat the weak measurement as a powerful tool for addressing the counterintuitive quantum phenomena seen in Refs. [8 12]. Thus, the weak value is predetermined, and it corresponds to the so-called fixed value. In this case, the measurement backaction can be ignored by setting a sufficiently small coupling constant.

First, we calculate the spatial displacement of the probe state in the $x-y$ plane. In this case, we employ Eq. (6) up to the first order and obtain the spatial displacements as

$$
\begin{aligned}
& \langle\hat{X}\rangle_{\mathrm{fi}}=g\left(\operatorname{Re}\langle\hat{A}\rangle_{\mathrm{w}}+l \operatorname{Im}\langle\hat{B}\rangle_{\mathrm{w}}\right), \\
& \langle\hat{Y}\rangle_{\mathrm{fi}}=g\left(\operatorname{Re}\langle\hat{B}\rangle_{\mathrm{w}}-l \operatorname{Im}\langle\hat{A}\rangle_{\mathrm{w}}\right) .
\end{aligned}
$$

These results show that the spatial displacements along the $x$ and $y$ directions include not only the real part of the weak values but also its imaginary part except for $l=0$. This is because the probe state given in Eq. (2) is not factorable in the $x$ and $y$ dimensions so the coupling of the observable $\hat{A}(\hat{B})$ with the $x(y)$ dimension of the probe also affects its $y(x)$ dimension as the imaginary part of the weak value.

Next, we consider the joint measurement of two position observables along the mutually-perpendicular directions in the probe state. The joint observables in the 
probe state can be represented by $\hat{X} \hat{Y}$ and $\hat{X}^{2}-\hat{Y}^{2}$. The other products of the perpendicular position operators can be represented by the linear superposition of these two observables. The expectation values of these joint observables can be experimentally obtained from the two-dimensional intensity distribution captured by the imaging sensor.

We employ Eq. (6) up to the second order in the coupling constant $g$ and obtain the second-order spatial displacements as

$$
\begin{gathered}
\langle\hat{X} \hat{Y}\rangle_{\mathrm{fi}}=g^{2}\left[-\frac{l^{2}-|l|-2}{4} \operatorname{Re}\left(\langle\hat{A}\rangle_{\mathrm{w}}\langle\hat{B}\rangle_{\mathrm{w}}^{*}\right)+\frac{l^{2}-|l|+2}{4} \operatorname{Re}\left\langle\frac{\hat{A} \hat{B}+\hat{B} \hat{A}}{2}\right\rangle_{\mathrm{w}}-l \operatorname{Im}\left\langle\frac{\hat{A}^{2}-\hat{B}^{2}}{2}\right\rangle_{\mathrm{w}}\right], \\
\left\langle\frac{\hat{X}^{2}-\hat{Y}^{2}}{2}\right\rangle_{\mathrm{fi}}=g^{2}\left[-\frac{l^{2}-|l|-2}{8}\left(\left|\langle\hat{A}\rangle_{\mathrm{w}}\right|^{2}-\left|\langle\hat{B}\rangle_{\mathrm{w}}\right|^{2}\right)+\frac{l^{2}-|l|+2}{4} \operatorname{Re}\left\langle\frac{\hat{A}^{2}-\hat{B}^{2}}{2}\right\rangle_{\mathrm{w}}+l \operatorname{Im}\left\langle\frac{\hat{A} \hat{B}+\hat{B} \hat{A}}{2}\right\rangle_{\mathrm{w}}\right] .
\end{gathered}
$$

For the fundamental Gaussian probe state $(l=0)$, our result is consistent with the result given in Ref. [2]. In this case, Eqs. (11) and (12) contain only the real part of the joint weak values $\langle\hat{A} \hat{B}+\hat{B} \hat{A}\rangle_{\mathrm{w}}$ and $\left\langle\hat{A}^{2}-\hat{B}^{2}\right\rangle_{\mathrm{w}}$. For $|l|>0$, however, we obtain additional terms proportional to their imaginary part as illustrated in Ref. [3] for the case of $|l|=1$ [26]. Thus, the second-order spatial displacements contain all the information about joint weak values. If there are a sufficient number of different outcomes for the first- and second-order spatial displacements, we can calculate the joint weak values from Eqs. (9) - (12) [27]. This feature is not attainable with a fundamental Gaussian mode $(l=0)$.

One useful method for obtaining the joint weak values is to take two separate measurements by using two probe states with different $l$ values. From a practical point of view, the best choice is two probe states with equal magnitude but different signs of $l$ since they can be easily prepared by using a mirror reflection. In this method, the two separate measurements with four types of spatial displacements bring eight outcomes, which equals to the number of unknown real and imaginary parts of single and joint weak values. Thus, it is possible to calculate the real and imaginary parts of the joint weak values from Eqs. (9)- (12). The advantage of this method is that we can use the same interaction Hamiltonian, i.e., the same experimental setup; we only need to change the input probe state.

In particular, in case of $|l|=2$, the coefficients of the first-order weak values $\langle\hat{A}\rangle_{\mathrm{w}}$ and $\langle\hat{B}\rangle_{\mathrm{w}}$ in Eqs. (11) and (12) are eliminated. Therefore, only the joint weak values remain. By using the two probe states with $l= \pm 2$, we can extract the joint weak values only from the second- order spatial displacement as follows:

$$
\begin{aligned}
\operatorname{Re}\langle\hat{A} \hat{B}+\hat{B} \hat{A}\rangle_{\mathrm{w}} & =\frac{\langle\hat{X} \hat{Y}\rangle_{+}+\langle\hat{X} \hat{Y}\rangle_{-}}{g^{2}}, \\
\operatorname{Im}\langle\hat{A} \hat{B}+\hat{B} \hat{A}\rangle_{\mathrm{w}} & =\frac{\left\langle\hat{X}^{2}-\hat{Y}^{2}\right\rangle_{+}-\left\langle\hat{X}^{2}-\hat{Y}^{2}\right\rangle_{-}}{4 g^{2}}, \\
\operatorname{Re}\left\langle\hat{A}^{2}-\hat{B}^{2}\right\rangle_{\mathrm{w}} & =\frac{\left\langle\hat{X}^{2}-\hat{Y}^{2}\right\rangle_{+}+\left\langle\hat{X}^{2}-\hat{Y}^{2}\right\rangle_{-}}{2 g^{2}}, \\
\operatorname{Im}\left\langle\hat{A}^{2}-\hat{B}^{2}\right\rangle_{\mathrm{w}} & =-\frac{\langle\hat{X} \hat{Y}\rangle_{+}-\langle\hat{X} \hat{Y}\rangle_{-}}{2 g^{2}}
\end{aligned}
$$

where $\langle\cdot\rangle_{+}$and $\langle\cdot\rangle_{-}$correspond to the spatial displacements for the $l=+2$ and -2 cases, respectively.

Moreover, under the assumption of $\hat{A}^{2}=\hat{B}^{2}$, the joint weak value $\left\langle\hat{A}^{2}-\hat{B}^{2}\right\rangle_{\mathrm{w}}$ is also eliminated. Thus, we can derive a simple and direct relationship between the second-order spatial displacements and the joint weak value as

$$
\begin{aligned}
& \operatorname{Re}\langle\hat{A} \hat{B}+\hat{B} \hat{A}\rangle_{\mathrm{w}}=\frac{2}{g^{2}}\langle\hat{X} \hat{Y}\rangle_{\mathrm{fi}}, \\
& \operatorname{Im}\langle\hat{A} \hat{B}+\hat{B} \hat{A}\rangle_{\mathrm{w}}=\frac{\operatorname{sgn}(l)}{2 g^{2}}\left\langle\hat{X}^{2}-\hat{Y}^{2}\right\rangle_{\mathrm{fi}}
\end{aligned}
$$

With these equations, the joint weak value can be directly extracted from the spatial displacements by using a single LG probe state with $|l|=2$. Although our consideration is restricted only to the case of $\hat{A}^{2}=\hat{B}^{2}$, this case includes many experimental setups. For example, the joint weak measurement of Pauli operators $\hat{\sigma}_{i}(i=x, y, z)$ on different two-level systems, such as $\hat{A}=\hat{\sigma}_{z} \otimes \hat{I}$ and $\hat{B}=\hat{I} \otimes \hat{\sigma}_{z}$ with the identity operator $\hat{I}$, is included since they satisfy the property $\hat{A}^{2}=\hat{B}^{2}=\hat{I}$.

In this Rapid Communication, we derive the full description of the weak measurement for the LG probe state and provide a simple method for extracting the joint weak values only from the spatial displacements of the two-dimensional probe state. Our method has a statistical advantage over previous ones since information about the momentum displacement is not required. Moreover, by using the LG probe state with $|l|=2$, and for the case 
$\hat{A}^{2}=\hat{B}^{2}$, we can extract the full joint weak value, i.e., the real and imaginary parts of the joint weak value directly from the second-order spatial displacements without any additional data processing.

In our study, we restrict the probe state to the LG mode with the radial index $p=0$. However, we can use other spatial modes, e.g. LG modes with nonzero radial index $p$, Hermite Gauss modes, and hypergeometricGaussian modes. There remains an interesting problem on the relationship between the spatial rotational symmetry of the probe state and the joint weak value since the spatial rotational symmetry in our restricted case is broken after the weak measurement. Moreover, it would be interesting to study the case of probe states given by superpositions of different spatial modes. By using such a extended probe state, the condition $\hat{A}^{2}=\hat{B}^{2}$ might be relaxed.

We thank Y. Aharonov, J. Tollaksen, A. M. Steinberg, and L. Vaidman for useful comments and suggestions. One of the authors (GP) acknowledges financial support from the Marie Curie Incoming Fellowship COFUND.
[1] Q. A. Turchette, C. J. Hood, W. Lange, H. Mabuchi, and H. J. Kimble, Phys. Rev. Lett. 75, 4710 (1995); J. D. Franson, ibid. 78, 3852 (1997).

[2] K. J. Resch and A. M. Steinberg, Phys. Rev. Lett. 92, 130402 (2004).

[3] G. Puentes, N. Hermosa, and J. P. Torres, Phys. Rev. Lett. 109, 040401 (2012).

[4] G. Mitchison, R. Jozsa, and S. Popescu, Phys. Rev. A 76, 062105 (2007).

[5] Y. Aharonov, A. Botero, S. Popescu, B. Reznik, and J. Tollaksen, Phys. Lett. A 301, 130 (2002); A. Hosoya and Y. Shikano, J. Phys. A 43, 385307 (2010).

[6] Y. Aharonov, D. Z. Albert, and L. Vaidman, Phys. Rev. Lett. 60, 1351 (1988).

[7] Y. Aharonov, P. G. Bergmann, and J. L. Lebowitz, Phys. Rev. 134, B1410 (1964).

[8] J. S. Lundeen, B. Sutherland, A. Patel, C. Stewart, and C. Bamber, Nature (London) 474, 188 (2011).

[9] S. Kocsis, B. Braverman, S. Ravets, M. J. Stevens, R. P. Mirin, L. K. Shalm, and A. M. Steinberg, Science 332, 1170 (2011).

[10] L. A. Rozema, A. Darabi, D. H. Mahler, A. Hayat, Y. Soudagar, and A. M. Steinberg, Phys. Rev. Lett. 109, 100404 (2012)

[11] J. S. Lundeen and A. M. Steinberg, Phys. Rev. Lett. 102, 020404 (2009); K. Yokota, T. Yamamoto, M. Koashi, and N. Imoto, New J. Phys. 11, 033011 (2009).

[12] A. Palacios-Laloy, F. Mallet, F. Nguyen, P. Bertet, D. Vion, D. Esteve, and A. N. Korotov, Nat. Phys. 6, 442 (2010); J. Dressel, C. J. Broadbent, J. C. Howell, and A. N. Jordan, Phys. Rev. Lett. 106, 040402 (2011); M. E.Goggin, M. P. Almeida, M. Barbieri, B. P. Lanyon, J. L. OBrien, A. G. White, and G. J. Pryde, Proc. Natl. Acad. Sci. U.S.A. 108, 1256 (2011); Y. Suzuki, M. Iinuma, and H. F. Hofmann, arXiv:1206.6954.

[13] H. Kobayashi, S. Tamate, T. Nakanishi, K. Sugiyama, and M. Kitano, J. Phys. Soc. Jpn. 80, 034401 (2011).

[14] Y. Shikano, in Measurements in Quantum Mechanics, edited by M. R. Pahlavani, (InTech, Rijeka, Croatia, 2012), Chap. 4, p. 75

[15] Y. Aharonov and L. Vaidman, in Time in Quantum Mechanics, Vol. 1, edited by J. G. Muga, R. Sala Mayato, and I. L. Egusquiza (Springer, Berlin, 2002), p. 399.

[16] Y. Aharonov and J. Tollaksen, in Visions of Discovery: New Light on Physics Cosmology and Consciousness, edited by R. Y. Chiao, M. L. Cohen, A. J. Leggett, W. D. Phillips, and C. L. Harper, Jr. (Cambridge University Press, Cambridge, 2011), p. 105.

[17] R. Jozsa, Phys. Rev. A 76, 044103 (2007).

[18] A. Siegman, Lasers, (University Science Books, Sausalito, CA, 1986).

[19] L. Allen, M. W. Beijersbergen, R. J. C. Spreeuw, and J. P. Woerdman, Phys. Rev. A 45, 8185 (1992).

[20] T. Ando, Y. Ohtake, N. Matsumoto, T. Inoue, and N. Fukuchi, Opt. Lett. 34, 34 (2009).

[21] H. Kobayashi, K. Nonaka, and M. Kitano, Opt. Express 20, 14064 (2012).

[22] N. B. Simpson, L. Allen, and M. J. Padgett, J. Mod. Opt. 43, 2485 (1996).

[23] B. Mours, E. Tournefier, and J.-Y. Vinet, Class. Quantum Grav. 23, 5777 (2006).

[24] A. Mair, A. Vaziri, G. Weihs, and A. Zeilinger, Nature (London) 412, 313 (2001).

[25] S. Wu and Y. Li, Phys. Rev. A 83, 052106 (2011); J. P. Torres, G. Puentes, N. Hermosa, and L. J. SalazarSerrano, Opt. Express 20, 18869 (2012).

[26] Equation (11) includes a correction for a typographical error in Eq. (4) in Ref. [3].

[27] It is noted that one can compute $\langle\hat{A} \hat{B}+\hat{B} \hat{A}\rangle_{\mathrm{w}}$ but not $\langle\hat{A} \hat{B}\rangle_{\mathrm{w}}$ or $\langle\hat{B} \hat{A}\rangle_{\mathrm{w}}$ except for $[\hat{A}, \hat{B}]=c \hat{I}$, where $c$ is the imaginary number and $\hat{I}$ is the identity operator. 